\title{
Recent trends in the incidence of recorded depression in primary care
}

\author{
Greta Rait, Kate Walters, Mark Griffin, Marta Buszewicz, Irene Petersen and Irwin Nazareth
}

\section{Background}

There is a paucity of data describing how general practitioners (GPS) label or record depression.

\section{Aims \\ To determine incidence and sociodemographic variation in GP-recorded depression diagnoses and depressive symptoms.}

\section{Method}

Annual incidence rates calculated using data from 298 UK general practices between 1996 and 2006, adjusted for year of diagnosis, gender, age and deprivation.

\section{Results}

Incidence of diagnosed depression fell from 22.5 to 14.0 per 1000 person-years at risk (PYAR) from 1996 to 2006. The incidence of depressive symptoms rose threefold from 5.1 to 15.5 per 1000 PYAR. Combined incidence of diagnoses and symptoms remained stable. Diagnosed depression and symptoms were more common in women and in more deprived areas.

\section{Conclusions}

Depression recorded by general practitioners has lower incidence rates than depression recorded in epidemiological studies, although there are similar associations with gender and deprivation. General practitioners increasingly use symptoms rather than diagnostic labels to categorise people's illnesses. Studies using standardised diagnostic instruments may not be easily comparable with clinical practice.

\section{Declaration of interest}

None.
Depression will be the second highest cause of disease burden globally by $2020 .^{1}$ The cost of depression has recently been estimated at $\$ 83.1$ billion in the $\mathrm{USA}^{2}$ and $£ 9$ billion in England, ${ }^{3}$ most of which was attributable to time off work. Epidemiological surveys suggest that the prevalence of depression has risen in the USA, ${ }^{4,5}$ although UK survey data has not shown similar changes. ${ }^{6}$ Antidepressant prescribing has risen in the USA and UK over the past decade. $^{3,7}$ Data from six European countries ${ }^{8}$ reported prevalence rates of $13.9 \%$ in women and $8.5 \%$ in men of DSM-IV major depression in family practice attendees in 2004-2005.

The diagnostic validity of depression is a cause of much debate, and increasingly the concept is being questioned. Psychiatric research classifies psychological disorders according to defined criteria, such as those in the DSM-IV ${ }^{9}$ or ICD-10. ${ }^{10}$ However, primary care depression may be described as an everyday problem of practice, rather than an objective diagnostic category. ${ }^{11}$ Standardised diagnostic interviews are not used in primary care to diagnose depression, and it is known that more severe depression is more likely to be recognised in primary care settings. ${ }^{12,13}$ A recent meta-analysis ${ }^{14}$ found that non-psychiatric physicians' recognition of depression had a low sensitivity (36.4\%) but high specificity ( $83.7 \%$ ) for 'true' cases measured using standardised diagnostic instruments; however, non-psychiatric physicians may deliberately avoid labelling people with mild symptoms as being depressed. Therefore, categorical approaches to diagnosis have been questioned in favour of a multidimensional approach, ${ }^{15}$ which may be more relevant to primary care. ${ }^{12}$

Epidemiological surveys have shown that depression diagnoses are higher in women, older people and those living in deprived areas. We do not know whether this is mirrored in the rates of general-practitioner (GP) recording of depression diagnoses and depressive symptoms. There are few data on depression incidence in primary care and primary care recording of depression, in contrast to the wealth of epidemiological data on prevalence. ${ }^{16}$ We examined a large UK primary care database to determine the incidence rates of recorded depression diagnoses and depressive symptoms in attenders from 1996 to 2006. We also determined whether there were differences in incidence of recording of diagnoses or symptoms by sociodemographic factors. The study was given a favourable opinion by the London Medical Research Ethics Committee (07/MRE02/5).

\section{Method}

\section{Setting}

We used data from 298 UK general practices, providing two or more years of data to The Health Improvement Network (THIN) from January 1996 to March 2006. We only used practices meeting standards for acceptable levels of data recording. The total registered patient population during this time period was 4986111 people, comprising 21.6 million years of follow-up.

\section{Data source}

The Health Improvement Network (THIN) electronic recording scheme is one of the largest UK sources of continuous primary care data on illness recording and prescribing behaviour (http:// www.thin-uk.com/). It has been used for epidemiological studies, ${ }^{17,18}$ including work on antidepressants and suicide. ${ }^{19}$ Anonymised patient data are pre-collected from participating practices. Practices are broadly representative of UK general practices in terms of patients' age and gender, practice size and geographical distribution. ${ }^{20}$ General practitioners enter medical diagnoses and symptoms using Read codes. Read codes are a hierarchical recording system used to record clinical summary information. The codes are not limited to diagnostic and procedural codes, but also include codes for symptoms, test results, screening and other areas. The age, gender, medical diagnosis and symptom records, health promotion activity, secondary care referrals, prescriptions and quintiles of deprivation score are recorded for each registered individual. 


\section{Study population}

All adults aged 16 years and over registered with practices providing acceptable levels of recording during the study period (1996-2006) were included in the study. All participants had a minimum of 1-year follow-up data.

\section{Measurements}

Read code lists were developed by the study team (Online supplements 1 and 2) to identify either recorded diagnoses of depression (e.g. 'depressive disorder') or recorded depressive symptoms (e.g. 'low mood'). A new episode of diagnosed depression was defined as an entry in the records where there was no previous diagnosis of depression coded in the previous year. A new episode of depressive symptoms was also defined as an entry where there had been no previous recorded depressive symptom code in the previous year. Participants could have more than one new episode within the study follow-up period, providing there were 12 months between episodes.

We excluded data from the first 12 months following registration with their GP, as this may be retrospective recording of a past history rather than a true incident recording of a new episode of depression. ${ }^{21}$ Age was examined by five age bands (16-24, 25-44, 45-64, 65-74, 75 and above). Deprivation was examined using quintiles of Townsend score from 'one' (least deprived) to 'five' (most deprived). The Townsend score ${ }^{22}$ is a combined measure of owner-occupation, car ownership, overcrowding and unemployment based on a patient's postcode and linkage to population census data for 2001 for approximately 150 households in that postal area.

\section{Statistical analysis}

Cases were defined using the Read codes for depression diagnosis and depressive symptoms. Annual incidence rates were calculated by dividing the annual number of incident cases by the total person-years at risk (PYAR) for each year. Single variable analysis was undertaken to examine the association between year of diagnosis (1996 to 2006), gender, age group and quintiles of deprivation score on the one hand and incidence of recorded depression diagnosis or depressive symptoms on the other. Poisson regression was undertaken to investigate the adjusted associations between incidences of recorded depression and depressive symptoms and year of diagnosis, gender, age group and deprivation. The significance of variables in the Poisson regression modelling was assessed using Wald tests. Robust standard errors for the estimated incidence rate ratios (IRRs) were utilised to account for clustering within practices. We examined whether there were interactions between age, gender, deprivation and calendar year. Analysis was conducted using Stata version 10 for Windows.

\section{Results}

\section{Sample characteristics}

There were 2982024 registered patients aged 16 years and over from January 1996 to March 2006. The number of incident recorded episodes of diagnosed depression was 255667, and of depressive symptoms 156907 . The median follow-up time was 4.9 years (10th centile 1.6 , 90th centile 11.8) for all patients, 6.2 years (10th centile 2.1, 90th centile 14.2) for those with a recorded depression diagnosis and 6.3 (10th centile 2.1, 90th centile 13.2) for those with recorded depressive symptoms.

The most frequently used codes for diagnosis were nonspecific codes: 'depressive disorder NEC' (not elsewhere classified) ( $n=90928$ episodes, $35.6 \%)$ and 'depression NOS' (not otherwise specified) ( $n=46681$ episodes, $18.2 \%$ ). The most common symptom codes were 'depressed' $(n=46071,29.4 \%)$ and 'complaining of feeling depressed' ( $n=45203,28.8 \%$ ).

Owing to the large sample size (events and PYAR) all associations were found to be statistically highly significant $(P<0.0001)$. The magnitude of the IRRs and associated 95\% confidence intervals (CIs) were therefore of primary interest in interpreting any associations.

\section{Incidence of recorded depression diagnoses}

The incidence of recorded diagnoses fell from 22.5/1000 PYAR in 1996 to $14.0 / 1000$ PYAR in 2006 (IRR $=0.64,95 \%$ CI $0.57-0.71$; online Table DS1). Females were more than twice as likely to have a diagnosis recorded than males. People in the 25 to 44 age group had the highest rate of diagnosed depression, their incidence being more than 50\% higher than those in the 16 to 24 age group. People in the most deprived group had nearly twice the rate of depression diagnosis compared with the least deprived group. The effect of gender and deprivation on incidence of depression did not change substantially with time (online Table DS1).

There was an interaction of age with calendar year, and age with gender. Therefore the IRRs for year, compared with the baseline of 1996, were stratified by both age and gender (Tables 1 and 2). The incidence of recorded depression over time fell more markedly in older (aged 65 years or more) compared with younger age groups. For example, in 1996 the incidence of diagnosed depression in those aged 75 and over was $32 \%$ greater than that for 16- to 24-year-olds. By 2006 this was reversed with the incidence in those aged 75 and over being 23\% lower. In females the overall incidence of depression in those aged 75 and over was $16 \%$ less than in the 16- to 24 -year-olds, whereas in males there was a $43 \%$ increase.

\section{Incidence of recorded depressive symptoms}

The incidence of recorded depressive symptoms rose threefold from the baseline of 5.11/1000 PYAR in 1996 to $15.5 / 1000$ PYAR in 2006 (online Table DS2). Females had symptoms recorded twice as often as males, which is similar to the findings of recorded diagnoses. Adults in the 25 to 44 age group had the highest rate of depression symptoms, compared with those in the 16 to 24 age group. Those in the most deprived group had depressive symptoms recorded nearly twice as often as the least deprived group.

Table 1 Incidence rate ratios (IRR) for diagnosed

depression and symptoms of depression stratified by year

\begin{tabular}{|c|c|c|}
\hline $\begin{array}{l}\text { Multivariable: } \\
\text { by year, }\end{array}$ & & $\begin{array}{c}\text { Multivariable: }{ }^{\text {a }} \text { stratified } \\
\text { by year, } 2006\end{array}$ \\
\hline IRR (ৎ) & $P^{\mathrm{b}}$ & IRR (95\% Cl) \\
\hline
\end{tabular}

Diagnosed depression

Age group

$\begin{array}{lcccc}16-24 & \text { Baseline } & <0.0001 & \text { Baseline } & <0.0001 \\ 25-44 & 1.77(1.62-1.93) & 1.43(1.27-1.61) & \\ 45-64 & 1.67(1.52-1.82) & 1.31(1.15-1.49) & \\ 65-74 & 1.20(1.07-1.33) & 0.81(0.70-0.94) & \\ 75+ & 1.32(1.17-1.49) & 0.77(0.66-0.90) & \end{array}$

symptoms of depression

Age group

$16-24$

25-44

$45-64$

$<0.0001$

$65-74$

$1.78(1.54-2.06)$

$1.53(1.31-1.79)$

$75+$

$1.28(1.07-1.53)$

$10(1.00-1.21)$

$0.97(0.87-1.07)$

$0.62(0.54-0.71)$

$0.68(0.59-0.78)$

a. Adjusted for calendar year, gender, age, deprivation, and for clustering by general practitioner practice using robust standard errors.

b. $P$ based on Wald test. 
Table 2 Incidence rate ratios (IRR) for diagnosed

depression and symptoms of depression stratified by gender

\begin{tabular}{|c|c|c|}
\hline $\begin{array}{l}\text { Multivariable: } \\
\text { by gender }\end{array}$ & & $\begin{array}{l}\text { Multivariable } \\
\text { by gende }\end{array}$ \\
\hline IRR & $P^{\mathrm{b}}$ & IRR (95\% Cl) \\
\hline
\end{tabular}

$\begin{array}{lccc}\begin{array}{l}\text { Diagnosed depression } \\ \text { Age group }\end{array} & & \\ 16-24 & \text { Baseline } & <0.0001 & \text { Baseline } \\ 25-44 & 1.61(1.53-1.69) & 1.55(1.47-1.63) \\ 45-64 & 1.71(1.61-1.81) & 1.28(1.20-1.35) \\ 65-74 & 1.10(1.03-1.17) & 0.83(0.77-0.89) \\ 75+ & 1.43(1.32-1.54) & 0.84(0.78-0.91)\end{array}$

symptoms of depression

Age group

16-24 Baseline $\quad<0.0001 \quad$ Baseline $<0.0001$

25-44 $\quad 1.35(1.29-1.41) \quad 1.33(1.28-1.39)$

45-64 $1.38(1.32-1.46) \quad 1.11(1.06-1.17)$

65-74 $0.97(0.90-1.03) \quad 0.78(0.73-0.84)$

$75+\quad 1.36(1.25-1.48) \quad 0.85(0.79-0.92)$

a. Adjusted for calendar year, gender, age, deprivation, and for clustering by genera practitioner practice using robust standard errors.

b. $P$ based on Wald test.

For recorded depressive symptoms there was an interaction of age with calendar year and age with gender, so the IRRs for year, compared with the baseline of 1996, were stratified by age and gender (Tables 1 and 2). In 1996 the incidence of depressive symptoms in those aged 75 and over was $41 \%$ greater than in the 16- to 24-year-olds, whereas in 2006 the incidence was 32\% less. There was a lower incidence of recorded depressive symptoms in older age groups (aged 65 years or more) compared with younger age groups in women, but this difference was less marked among men. The effect of gender and deprivation on incidence of symptoms did not change substantially with time.

\section{Combined incidence for diagnoses and symptoms}

The results above demonstrate a fall in the recorded incidence of diagnosed depression, but an increase in recorded depressive symptoms, although the combined incidence rates varied little over time (Fig. 1). The combined incidence over the study period was 24.8/1000 PYAR, equivalent to a mean annual incidence of $2.5 \%$. We had categorised depression codes into two lists (diagnoses and symptoms) and included the code 'depressed' in the symptoms list. However, this code is commonly used and may be used by GPs as a diagnostic code, so we re-analysed the data with 'depressed' as a diagnostic code. This made only a small difference, slightly increasing the incidence of diagnosis, and

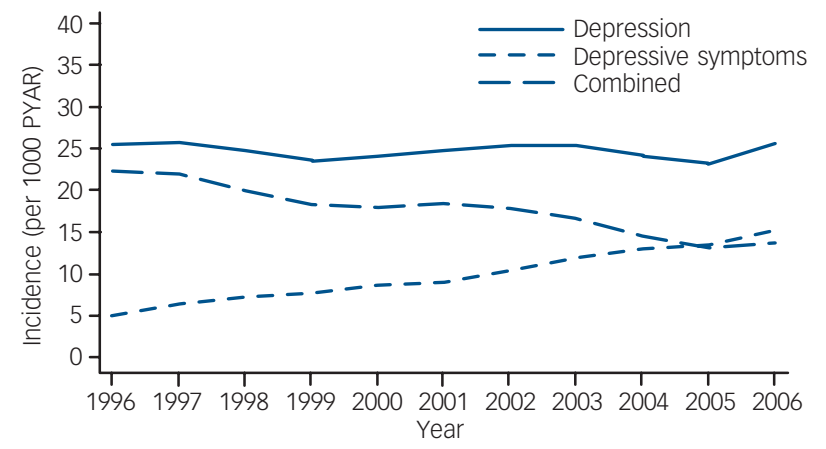

Fig. 1 Incidence of diagnosed depression and depressive symptoms.

PYAR, person-years at risk. decreasing the incidence of symptoms, and made no appreciable difference to the IRRs.

\section{Discussion}

\section{Key findings}

Since 1996 the incidence of recorded diagnoses of depression in UK general practice has fallen, whereas the incidence of recorded depressive symptoms has increased. However, the combined overall incidence has remained remarkably stable. The incidence, for both diagnosis and symptoms, was highest in females and those in the most deprived quintiles of the population. This finding was consistent over time. People in the 25- to 44- and 45to 64-year-old age groups had the highest incidence of both depression diagnoses and depressive symptoms. Recording of depression in older adults seems to have fallen relative to younger groups.

Most primary care research is based on community samples or on GP attendees, with a predominant focus on prevalence. Our study reports on people who had consulted GPs and been identified as having depression or depressive symptoms, and this information had then been entered in the electronic record. We would therefore expect lower incident rates than those observed in epidemiological case-finding studies of community or GP samples. To our knowledge there are few studies that have reported on this population and the GPs' entry of diagnosis in the electronic records.

The decrease in recorded depression diagnoses in our study contrasts with the increasing prevalence of the disorder in USA epidemiological studies, ${ }^{4,5}$ although UK community studies ${ }^{6}$ indicate that the prevalence of 'neurotic disorders' has remained stable despite rising antidepressant prescriptions. ${ }^{7,19}$ Our amalgamated incidence rates of depression diagnoses and symptoms remained stable over the 10-year period, which mirrors similar community prevalence rates recorded in the two waves of the British Survey of Psychiatric Morbidity ${ }^{6}$ (1993 and 2000).

Our results could be explained by a change in GPs' recording behaviour, with a reduced entry of depression as a diagnosis and an increased recording of depressive symptoms during this period, rather than a true decrease in the incidence of depression. The move towards recording symptoms and less specific terms may be perceived as less stigmatising for individuals. General practitioners' coding may be linked to the perceived severity of depression, with symptom codes being used for milder depression. It may also be explained by greater questioning of the meaning of psychiatric diagnostic categories in primary care ${ }^{23}$ and a move away from the use of discrete categories that have dominated psychiatric research and practice. General practitioners may be increasingly unhappy to label people with distress and low mood as having 'depressive disorder', and with the increased use of computer coding for consultations have started to enter symptom codes instead. General practitioners have a relationship with their patients that often results in diagnosis being made in a longitudinal manner. ${ }^{24}$ General practitioners take into account many psychosocial variables, but are also influenced by their own attitudes and the consulting environment, particularly time issues. ${ }^{25,26}$ Diagnostic validity remains a challenge. There is a continuing debate around whether GPs underdiagnose depression, when compared with psychiatric instruments, or whether they are recognising 'true' depression, and not medicalising normal human distress. ${ }^{11,23}$

The relationships between recorded depression diagnoses and symptoms, and gender and deprivation observed in this study are consistent with epidemiological research. ${ }^{27}$ The highest incidence rates in our study were in the 25- to 44-year-old age group for women and in the 44- to 65-year-old age group for men. This 
compares with contemporary studies of GP populations where the highest prevalence of depression was in women aged 18 to 30, and men aged 30 to 50 years. ${ }^{8}$ Over the study period the relative incidence of depression diagnoses has remained twice as high in more deprived areas compared with least deprived areas. This inequality is marked and stable over time. The same findings were observed with regard to depressive symptoms.

\section{Comparison with other studies}

Our study looked at the incidence of recorded depression diagnosis or symptoms. We included people with single episodes, or recurrent episodes provided there was a year between episodes. There is limited comparable incidence data from other sources, as most data pertain to prevalence ${ }^{28}$ or are based on epidemiological studies using diagnostic case finding in the community or with screened practice attendees, rather than GP identification and recording.

Our study found an overall combined incident rate of recorded depression (diagnoses and symptoms) of $2.5 \%$. This is lower than incidences found in epidemiological studies, which use case-finding and are limited to major depressive disorder. For example, in the PREDICT study in six European countries, ${ }^{29}$ the 12-month cumulative incidence of DSM-IV major depression in screened GP attendees was $7.7 \%$, and in the UK was $8.8 \%$. A systematic review in 2004 of international incidence studies of mood disorders ${ }^{16}$ identified four community studies but no primary care studies. They calculated a best-estimate rate for 1-year community incidence of major depressive disorder of 2.9 per 100 PYAR. The World Health Organization study in 15 international centres ${ }^{30}$ found $4.4 \%$ with new diagnoses of ICD-10 depressive episodes. In one of the studies closest to ours in design, a retrospective USA cohort study ${ }^{31}$ conducted in 39 practices in 1996 looked at incidence of first-ever recorded depressive episode in primary care settings in electronic records, excluding people with any history of depression or its treatment. They found that $2103(1.6 \%)$ of the 131141 eligible patients had a first-ever episode of recorded depression over 1 year. Our study was not limited to first-ever episodes. There is evidence that GPs may not record depression that they recognise. In a two-phase observational GP study based in seven practices, ${ }^{32}$ which looked at GP identification and prescribing for depression, GPs discussed depression with about half of the patients that they considered to have depression.

\section{Strengths and limitations}

This is one of very few studies examining the incidence of recorded depression in a large cohort of people seen in primary care. It included data from over 200000 new episodes of diagnosed depression and over 150000 new episodes of depressive symptoms recorded in routine primary care settings. A 10-year period was examined and changes over time evaluated. We defined our episodes by problem codes (diagnosis/symptoms) as we were looking specifically at GP-recorded depression. People who received a prescription for an antidepressant without a depression code being entered were excluded, as it was unclear what the GP's working diagnosis was in such cases. This group would include those prescribed antidepressants for another indication, such as chronic pain, as well as unlabelled depression.

The data are limited to GP-recorded depression in practice attendees, which reflects incidence, presentation and recording at a practice level only. Our data do not include people with whom depression was discussed, but not entered as a problem onto their clinical record. It is therefore very likely that this is an underestimate of GP-recognised depression. There is also little indication of severity from the Read codes used. Most codes entered were non-specific, not allowing for differentiation of subtypes. There is a lack of validation work on depression and depressive symptoms recording in UK primary care databases, and we did not therefore have a gold-standard comparison. There is evidence that non-psychiatric physicians' recognition of depression has a low sensitivity but high specificity for 'true' cases measured using standardised instruments. ${ }^{14}$ As computers are used more in practice, GPs are coding more problems, but also using the non-coded free text options more. These comments may allude to recognised depression, but will not get picked up in a search, resulting in an underestimate of the incidence.

\section{Implications}

This study suggests that the way that GPs choose to record depression has changed over the past decade. General-practitioner recording of depression diagnoses is lower than that reported in studies on GP attendees using active case-finding. The categorisation by GPs, however, does reflect what is known about depression (being more common in women and in areas of greater deprivation). It reinforces the suggestion that GPs may often choose not to use formal psychiatric criteria to define people's illnesses. ${ }^{23}$ The findings of epidemiological studies, using standardised diagnostic instruments, may not easily be compared with routine clinical practice, as different diagnostic criteria are being applied. This again raises issues regarding the diagnostic validity of depression diagnoses.

Primary care is the place where most people with depression are managed, but the debate over the identification and treatment of depression continues. ${ }^{23}$ Epidemiologists and psychiatrists have called for the better identification and treatment of depression in light of its high reported prevalence and associated morbidity. ${ }^{33}$ However, the high lifetime prevalence of depression $^{34}$ may also suggest that in many cases depression is a normal part of life. ${ }^{23,35}$ This may be reflected in the ways in which GPs record their findings. Currently the evidence that the identification of depression in clinical practice leads to better long-term outcomes is contested. ${ }^{35,36}$

Clinical data-sets may become a valuable tool in examining depression and its management in primary care. However, further work on the validation of recorded diagnoses is required to allow appropriate exploitation of electronic records. The combined incidence of diagnoses and symptoms remained stable, indicating that GPs may have recorded similar disorders differently over time. Research needs to address why GPs are using symptom codes more than diagnostic codes. Our study pre-dates the introduction of incentive payments for GPs in April 2006 to record depression as part of the National Health Service's Quality and Outcomes Framework (QoF), ${ }^{37}$ which may have further altered coding. Future work should examine the impact of the recommended use of psychiatric screening instruments on identification and classification of depression, as advocated in QoF.

\footnotetext{
Greta Rait, MRCGP, MD, MRC General Practice Research Framework, University College London Medical School; Kate Walters, MSC, MRCGP, Mark Griffin, MSC, Marta Buszewicz, MRCGP, MRCPsych, Irene Petersen, PhD, Research Department Marta Buszewicz, MRCGP, MRCPsych, Irene Petersen, PhD, Research Department
of Primary Care and Population Health, University College London Medical School, Irwin Nazareth, PhD, FRCGP, MRC General Practice Research Framework, University College London Medical School, London, UK

Correspondence: Dr Greta Rait, MRC General Practice Research Framework Stephenson House, 158-160 North Gower Street, London NW1 2ND, UK. Email: g.rait@pcps.ucl.ac.uk

First received 20 Aug 2008, final revision 22 Jun 2009, accepted 25 Jun 2009
} 


\section{Funding}

The Department of Primary Care and Population Health, University College London holds a license to conduct analysis on THIN. The Departmental THIN/GPRD Executive Committee reviewed and approved the study protocol, but had no involvement in the analysis, interpretation or decision to submit for publication. K.W. and I.P. were supported by Special Training Fellowship in Health Services Research from the Medical Research Council (UK). This sponsor had no involvement in the study design, data collection, analysis, interpretation or decision to submit for publication.

\section{Acknowledgement}

We would like to thank Amir Islam, data manager, who extracted the data.

\section{References}

1 Murray $\mathrm{CJ}$, Lopez $\mathrm{AD}$. Alternative projections of mortality and disability by cause 1990-2020: Global Burden of Disease Study. Lancet 1997; 349: 1498-504.

2 Greenberg P, Kessler R, Birnbaum H, Leong S, Lowe S, Berglund P, et al. The economic burden of depression in the United States: how did it change between 1990 and 2000? J Clin Psychiatry 2003; 64: 1465-75

3 Thomas CM, Morris S. Cost of depression among adults in England in 2000 Br J Psychiatry 2003; 183: 514-9.

4 Hasin D, Goodwin R, Stinson F, Grant B. Epidemiology of major depressive disorder results from the National Epidemiologic Survey on Alcoholism and Related Conditions. Arch Gen Psychiatry 2005; 62: 1097-106.

5 Kessler RC, Demler O, Frank RG, Olfson M, Pincus HA, Walters EE, et al. Prevalence and treatment of mental disorders, 1990 to 2003. New Engl J Med 2005; 352: 2515-23.

6 Singleton, N., Meltzer, H, Jenkins, R. Building a picture of psychiatric morbidity in a nation: a decade of epidemiological surveys in Great Britain. Int Rev Psychiatyr 2003; 15: 19-28.

7 Hollinghurst S, Kessler D, Peters T, Gunnell D. Opportunity costs of antidepressant prescribing in England: analysis of routine data. BMJ 2005; 330: 999-1000

8 King M, Nazareth I, Levy G, Walker C, Morris R, Weich S, et al. Prevalence of common mental disorders in general practice attendees across Europe. Br J Psychiatr 2008; 192: 362-7.

9 American Psychiatric Association. Diagnostic and Statistical Manual of Mental Disorder (4th edn) (DSM-IV). APA, 1994.

10 World Health Organization. The ICD-10 Classification of Mental and Behavioural Disorders: Clinical Descriptions and Diagnostic Guidelines. WHO, 1992.

11 Chew-Graham C, Mullin S, May C, Hedley S, Cole H. Managing depression in primary care: another example of the inverse care law? Fam Prac 2002; 19: $632-7$.

12 Thompson C, Ostler K, Peveler RC, Baker N, Kinmouth A-L. Dimensional perspective on the recognition of depressive symptoms in primary care. The Hampshire Depression Project 3. Br J Psychiatry 2001; 179: 317-23.

13 Goldberg D, Privett M, Ustun B, Simon G, Linden M. The effects of detection and treatment on the outcome of major depression in primary care: a naturalistic study in 15 cities. Brit J Gen Pract 1998; 48: 1840-4.

14 Cepoiu M, McCusker J, Cole MG, Sewitch M, Belzile E, Ciampi A. Recognition of depression by non-psychiatric physicians - a systematic literature review and meta-analysis. J Gen Intern Med 2008; 23: 25-36.

15 Das-Munshi J, Goldberg D, Bebbington PE, Bhugra DK, Brugha TS, Dewey ME, et al. Public health significance of mixed anxiety and depression: beyond current classification. Br J Psychiatry 2008; 192: 171-7.
16 Waraich P, Goldner EM, Somers JM, Hsu L. Prevalence and incidence studies of mood disorders: a systematic review of the literature. Can J Psychiatry 2004; 49: 124-38.

17 Bath-Hextall F, Leonardi-Bee J, Smith C, Meal A, Hubbard R. Trends in incidence of skin basal cell carcinoma. Additional evidence from a UK primary care database study. Int J Cancer 2007; 121: 2105-8.

18 Smeeth L, Cook C, Thomas S, Hall A J, Hubbard R, Vallance P. Risk of deep vein thrombosis and pulmonary embolism after acute infection in a community setting. Lancet 2006; 367: 1075-9.

19 Morgan O, Griffiths C, Majeed A. Antidepressant prescribing and changes in antidepressant poisoning mortality and suicide in England, 1993-2004. J Public Health 2008; 30: 60-8.

20 Bourke A, Dattani $H$, Robinson M. Feasibility study and methodology to create a quality evaluated database of primary care data. Inform Prim Care 2004; 12: 171-7.

21 Lewis JD, Bilker WB, Weinstein RB, Strom BL. The relationship between time since registration and measured incidence rates in the General Practice Research Database. Pharmacoepidem Dr S 2005; 14: 443-51.

22 Office of National Statistics. Census 2001. Office of National Statistics, 2001 (http://www.statistics.gov.uk/census2001/census2001.asp).

23 Dowrick C. Beyond Depression: A New Approach to Understanding and Management. Oxford Medical Publications, 2004.

24 Kessler D, Bennewith O, Lewis G, Sharp D. Detection of depression and anxiety in primary care: follow up study. BMJ 2002; 325: 1016-7.

25 Johnston O, Kumar S, Kendall K, Peveler R, Gabbay J, Kendrick T. Qualitative study of depression management in primary care: GP and patient goals, and the value of listening. Br J Gen Pract 2007; 57: 872-879.

26 Baik S, Bowers B, Oakley L, Susman J. The recognition of depression: the primary care clinician's perspective. Ann Fam Med 2005: 3: 31-7.

27 Lorant V, Deliege D, Eaton W, Robert A, Philippot P, Ansseau M. Socioeconomic inequalities in depression: a meta-analysis. Am J Epidemiol 2003; 157: 98-112.

28 WHO World Mental Health Survey Consortium. Prevalence, severity and unmet need for treatment of mental disorders in the World Health Organization World Mental Health Surveys. JAMA 2004; 291: 2581-90.

29 King M, Walker C, Levy G, Bottomley C, Royston P, weich S, et al. Development and validation of an international risk prediction algorithm for episodes of major depression in general practice attendees: the PREDICT study. Arch Gen Psychiatry 2008; 65: 1368-76.

30 Barkow K, Maier W, Ustun T, Gansicke M, Wittchen H, Heun R. Risk factors for new depressive episodes in primary health care: an international prospective 12 month study follow-up study. Psychol Med 2002; 32 595-607.

31 Ornstein S, Stuart G, Jenkins R. Depression diagnoses and antidepressant use in primary care practices. J Fam Pract 2000; 49: 68-72.

32 Kendrick T, King F, Albertella L, Smith PW. GP treatment decisions for patients with depression: an observational study. Br J Gen Pract 2005; 55 280-6.

33 Moussavi S, Chatterii S, Verdes E, Tandon A, Patel V, Ustun B. Depression chronic diseases, and decrements in health: results from the World Health Surveys. Lancet 2007; 370: 851-8.

34 Kessler R, Berglund P, Demler O, Jin R, Merikangas KR and Walters EE. Lifetime prevalence and age-of-onset distributions of DSM-IV disorders in the National Comorbidity Survey Replication. Arch Gen Psychiatry. 2005; 62: 593-602.

35 Heath I. There must be limits to the medicalisation of human distress BMJ 1999; 318: 439-40.

36 Parker G. Is depression overdiagnosed? Yes. BMJ 2007; 335: 328

37 Lester H, Sharp D, Hobbs FDR, Lakhani M. The Quality and Outcomes Framework of the GMS contract: a quiet evolution for 2006. Br J Gen Pract 2006; 56: 244-6. 